\title{
A CHARACTERIZATION OF CONTINUOUS DISTRIBUTIONS VIA REGRESSION ON PAIRS OF RECORD VALUES
}

\author{
I. Bairamov ${ }^{1}$, M. Ahsanullah ${ }^{2}$ and Anthony G. PaKes ${ }^{3 *}$ \\ Izmir University of Economics, University of South Florida and \\ The University of Western Australia
}

\begin{abstract}
Summary
The exponential type is characterized in terms of the regression of a (possibly non-linear) function of a record value with its adjacent record values as covariates. Monotone transformations extend this result to more general settings, and these are illustrated with some specific examples.
\end{abstract}

Key words: characterization; record values; regression.

\section{Introduction}

Let $X_{1}, X_{2}, \ldots$ be independent copies of a random variable $X$ whose distribution function is denoted by $F$. There have been many studies on characterizations of $F$ via linear regression relations of one order statistic on one or two other order statistics. See Wesolowski \& Ahsanullah (1997) for references and the most complete results. Similarly, the regression of one record value on another has received attention. Denote record times by $L(1)=1$ and, for $n>1$,

$$
L(n)=\min \left\{j: j>L(n-1) \text { and } X_{j}>X_{L(n-1)}\right\},
$$

and corresponding record values by $X(n)=X_{L(n)}$; see Nevzorov (2001).

Fix positive integers $n$ and $s$, and real constants $a$ and $b$. The one-variable linear regression problem is to determine all $F$ for which

$$
\mathrm{E}(X(n+s) \mid X(n)=x)=a x+b .
$$

Nagaraja (1977) first addressed this in the adjacent case $s=1$, showing that solutions exist if $a>0$ and that solutions comprise a unique type whose form depends on the value of $a$ relative to unity. Ahsanullah \& Wesolowski (1998) proved that the same solutions are determined in the case $s=2$. Finally Dembinska \& Wesolowski (2000) proved that the general case can be reduced to an application of the Lau-Rao theorem for solving the extended Cauchy equation. See Arnold et al. (1998 Section 4.4.2,3) for a compact account of the Lau-Rao theorem.

A formal extension of these results replaces (1) with $\mathrm{E}(h(X(n+s)) \mid X(n)=x)=a x+b$, where $h$ is a continuous and strictly monotonic function. In the case $s=1$ Franco \& Ruiz

Received February 2004; revised June 2004; accepted November 2004.

* Author to whom correspondence should be addressed.

${ }^{1}$ Dept of Mathematics, Izmir University of Economics, 35330 Balcova, Izmir, Turkey.

${ }^{2}$ Dept of Mathematics, University of South Florida, Tampa, Florida 33629, USA.

${ }^{3}$ School of Mathematics \& Statistics, The University of Western Australia, 35 Stirling Highway,

Crawley, WA 6009. e-mail: pakes@maths.uwa.edu.au 
(1996) investigate the deeper problem of determining $F$ from a specification of this function, which need not be linear. In the adjacent case, these problems, as well as order-statistics versions, can be transformed to characterization by the form of the conditional expectation of $X$ given truncation events such as $\{X \leq x\}$. These connections are reviewed by Pakes (2004) who shows how product integration yields simple solutions requiring very weak conditions, if any, on $F$.

In this paper we investigate the characterization of $F$ in terms of the bivariate regression function $\mathrm{E}\left(H(X(n)) \mid X(n-1)=u, X(n+1)=v\right.$ ) where $\ell_{F} \leq u<v \leq r_{F}$ and $\ell_{F}=$ $\inf \{x: F(x)>0\}$ and $r_{F}=\sup \{x: F(x)<1\}$, respectively, are the left and right extremities of $F$. Pakes (2004 Section 5) alludes briefly to this problem, but we aim to give an elementary treatment for the continuous case.

\section{A characterization of the exponential type}

Let $\bar{F}(x)=1-F(x)$ and, for $\ell_{F} \leq x<r_{F}$, let $B(x)=-\log \bar{F}(x)$. The Markov dependence of record values (Arnold, 1998 p. 28 or Nevzorov, 2001 p.68) can be used to show that

$$
\operatorname{Pr}(X(n) \in d x \mid X(n-1)=u, X(n+1)=v)=\frac{d B(x)}{B(v)-B(u)},
$$

whence

$$
\mathrm{E}(H(X(n)) \mid X(n-1)=u, X(n+1)=v)=\frac{1}{B(v)-B(u)} \int_{u}^{v} H(x) d B(x) .
$$

We draw two conclusions from this expression. First, if $F$ is the standard exponential distribution function then $B(x)=x(x \geq 0)$ and hence the right-hand side of (2) equals

$$
\frac{1}{v-u} \int_{u}^{v} H(x) d x=\frac{h(v)-h(u)}{v-u},
$$

where $h^{\prime}(x)=H(x)$. Second, if $h(x)=c x+b$, where $c$ and $b$ are real constants, then the right-hand side of (2) equals $c$ for any choice of $F$. Consequently, without loss of generality we can restrict attention to the regression relation

$$
\mathrm{E}\left(h^{\prime}(X(n)) \mid X(n-1)=u, X(n+1)=v\right)=\frac{h(v)-h(u)}{v-u} \quad\left(\ell_{F}<u<v<r_{F}\right),
$$

subject to a restriction on $h$ which excludes affine functions. Our first result does this under an additional restriction to absolutely continuous $F$. In the sequel, in any reference to (2) we understand that $H(x)=h^{\prime}(x)$.

Theorem 1. Suppose $F$ is absolutely continuous with density function $f$, that $h$ is continuous in $\left[\ell_{F}, r_{H}\right]$ and continuously differentiable in $\left(\ell_{F}, r_{F}\right)$, and that almost everywhere in this open interval,

$$
h^{\prime}(x) \neq \frac{h(x)-h_{1}}{x-\ell_{F}},
$$

where $h_{1}=h\left(\ell_{F}+\right)$. Then (3) holds if and only if $\ell_{F}>-\infty, r_{F}=\infty$ and

$$
F(x)=1-e^{-c\left(x-\ell_{F}\right)} \quad\left(x \geq \ell_{F}\right),
$$

where $c>0$ is an arbitrary constant. 
Proof. Equation (5) implies (3). For the converse, the continuity of $F$ entails $B\left(\ell_{F}\right)=0$, and hence letting $u \rightarrow \ell_{F}+$ in (3), it follows from (2) that

$$
\int_{\ell_{F}}^{v} h^{\prime}(x) B^{\prime}(x) d x=B(v) \frac{h(v)-h_{1}}{v-\ell_{F}} \quad\left(\ell_{F}<v<r_{F}\right) .
$$

Differentiating and re-arranging terms yields the identity

$$
\left(h^{\prime}(v)-\frac{h(v)-h_{1}}{v-\ell_{F}}\right) B^{\prime}(v)=\frac{B(v)}{v-\ell_{F}}\left(h^{\prime}(v)-\frac{h(v)-h_{1}}{v-\ell_{F}}\right) .
$$

The condition (4) implies that $B^{\prime}(v) / B(v)=1 /\left(v-\ell_{F}\right)$ and hence that $\bar{F}(v)=e^{-c\left(v-\ell_{F}\right)}$. It follows that $\ell_{F}>-\infty$ and $c>0$, and then the continuity of $F$ implies that $r_{F}=\infty$.

We conjecture that the absolute continuity assumption is unnecessary. Lemma 1 shows that we can transfer this assumption to a further smoothness assumption about $h$.

Lemma 1. If $h^{\prime \prime}(x)$ exists in $\left(\ell_{F}, r_{F}\right)$, and (3) and (4) both hold, then $F$ is absolutely continuous.

Proof. Again with $u=\ell_{F}$, integration by parts of the integral in (2) and re-arranging yields

$$
\int_{\ell_{F}}^{v} B(x) h^{\prime \prime}(x) d x=B(v)\left(h^{\prime}(v)-\frac{h(v)-h_{1}}{v-\ell_{F}}\right) .
$$

The integral is absolutely continuous and the coefficient of $B(v)$ is differentiable, and so it follows from the assumptions that $B$ is absolutely continuous.

Differing choices of $h$ yield many characterizations of the exponential type. One choice satisfying Lemma 1 is $h(x)=\frac{1}{2} x^{2}$.

Theorem 2. The continuous random variable $X$ has the exponential type (5) if and only if

$$
\mathrm{E}(X(n) \mid X(n-1)=u, X(n+1)=v)=\frac{1}{2}(u+v) \quad\left(\ell_{F}<u<v<\infty\right) .
$$

\section{Monotone transformations}

In this section we give a formal generalization of Theorem 1 which arises from monotone transformation of $X$. Specifically, we give a regression condition which specifies the form of the distribution function $G$ of a random variable $Y$. The corresponding record values are denoted by $Y(n)$.

Theorem 3. Suppose that:

(i) a random variable $Y$ has a continuous distribution function $G$ supported on $\left[\ell_{G}, r_{G}\right]$;

(ii) the function $R$ is continuous and strictly increasing in $\left(\ell_{G}, r_{G}\right)$ and

$$
\tau=R\left(\ell_{G}+\right)>-\infty \quad \text { and } \quad R\left(r_{G}\right)=\infty ;
$$

and

(iii) $h$ is twice differentiable in $(\ell, \infty)$. 
Then

$$
\begin{aligned}
\mathrm{E}\left(h^{\prime}(R(Y(n))) \mid Y(n-1)\right. & =s, Y(n+1)=t) \\
& =\frac{h(R(t))-h(R(s))}{R(t)-R(s)} \quad\left(\ell_{G}<s<t<r_{G}\right)
\end{aligned}
$$

if and only if

$$
G(y)=1-e^{-c(R(y)-\tau)} \quad\left(\ell_{G}<y<r_{G}\right),
$$

where $c>0$ is an arbitrary constant. If (ii) is replaced by

(ii') the function $R$ is continuous and strictly decreasing in $\left(\ell_{G}, r_{G}\right)$ and

$$
R\left(\ell_{G}+\right)=\infty \quad \text { and } \quad \tau=R\left(r_{G}\right) ;
$$

then (7) holds if and only if

$$
G(y)=e^{-c(R(y)-\tau)} \quad\left(\ell_{G}<y<r_{G}\right),
$$

where $c>0$ is an arbitrary constant.

Proof. If $X=R(Y)$ then the random variables $R(Y(j))(j=n-1, n, n+1)$ have the same joint distribution as $X(n-1), X(n), X(n+1)$. It follows that (7) is equivalent to (3) with $u=R(s)$ and $v=R(t)$. Theorem 1 and Lemma 1 imply that $R(Y)$ has an exponential type distribution (5) (with $\tau$ replacing $\ell_{F}$ ) and the assumptions about $R$ yield

$$
G(y)=P(R(Y) \leq R(y))=P(X \leq R(y)),
$$

whence (8). The argument is reversible. Equation (6) is necessary for the continuity of $G$. Altering some details shows that when (ii') replaces (ii) then (7) is equivalent to (9).

Combining Theorems 2 and 3 yields the following result which gives many special cases.

Theorem 4. If (i) and (ii) (resp. (ii')) of Theorem 3 hold then (8) (resp. (9)) holds if and only if

$$
\mathrm{E}(R(Y(n)) \mid Y(n-1)=s, Y(n+1)=t)=\frac{1}{2}(R(s)+R(t)) \quad\left(\ell_{G}<s<t<r_{G}\right) .
$$

Examples 1-3 follow from Theorem 4 with Condition (ii).

Example 1. If $\ell_{G}=0, r_{G}=\infty$, and $R(y)=y^{\alpha}$ for some constant $\alpha>0$, then $\tau=0$ and $Y$ has the Weibull distribution with $G(y)=1-\exp \left(-c y^{\alpha}\right)$ if and only if

$$
\mathrm{E}\left(Y^{\alpha}(n) \mid Y(n-1)=s, Y(n+1)=t\right)=\frac{1}{2}\left(s^{\alpha}+t^{\alpha}\right) \quad(0<s<t<\infty) .
$$

Example 2. If $\ell_{G}=0, r_{G}=1$ and $R(y)=-\log (1-y)$ then $\tau=0$ and $G(y)=1-(1-y)^{c}$ if and only if

$\mathrm{E}(\log (1-Y(n)) \mid Y(n-1)=s, Y(n+1)=t)=\frac{1}{2} \log ((1-s)(1-t)) \quad(0 \leq s<t<1)$.

Example 3. If $\ell_{G}=a>0, r_{G}=\infty$ and $R(y)=\log (y / a)$ then $\tau=0$ and $Y$ has the Pareto distribution with

$$
G(y)=1-(a / y)^{c} \quad(y \geq a)
$$

if and only if

$$
\mathrm{E}(\log Y(n) \mid Y(n-1)=s, Y(n+1)=t)=\log \sqrt{s t} \quad(a \leq s<t<\infty) .
$$

This is unexpected insofar as the form of the regression relation is independent of $a$. 
Examples 4 and 5 arise from Theorem 4 with Condition (ii' ${ }^{\prime}$ ).

Example 4. If $\ell_{G}=-\infty, r_{G}=\infty$, and $R(y)=e^{-y}$ then $\tau=0$ and $Y$ has the Gumbel extremal process marginal distribution function $G(y)=\exp \left(-c e^{-y}\right)$ if and only if

$$
\mathrm{E}\left(e^{-Y(n)} \mid Y(n-1)=s, Y(n+1)=t\right)=\frac{1}{2}\left(e^{-s}+e^{-t}\right) \quad(-\infty<s<t<\infty) .
$$

The case $c=1$ is the standard Gumbel distribution function $\Lambda(y)$.

Example 5. If $\ell_{G}=-\infty, r_{G}=\infty$, and $R(y)=\log \left(1+e^{-y}\right)$ then $\tau=0$ and $Y$ has a generalized logistic distribution, $G(y)=\left(1+e^{-y}\right)^{-c}$ if and only if

$$
\begin{aligned}
\mathrm{E}\left(\log \left(1+e^{-Y(n)}\right) \mid Y(n-1)\right. & =s, Y(n+1)=t) \\
& =\frac{1}{2} \log \left(\left(1+e^{-s}\right)\left(1+e^{-t}\right)\right) \quad(-\infty<s<t<\infty) .
\end{aligned}
$$

The constructions based on Condition (ii) are particular cases of the following. Suppose $A(y)$ is a continuous distribution function with support $\left[\ell_{G}, r_{G}\right]$, and strictly increasing therein, and if $R(y)=-\log \bar{A}(y)$ then

$$
G(y)=1-(1-A(y))^{c}
$$

for some $c>0$ if and only if (7) holds. Thus $A(y)=1-\exp \left(-y^{\alpha}\right)$ for Example 1, $y I_{[0,1]}(y)$ for Example 2, and $1-a / y$ for Example 3. Similarly, taking $R(y)=-\log A(y)$ yields $G(y)=(A(y))^{c}$ if and only if (7) holds. Thus $A(y)=\Lambda(y)$ for Example 4, and $A(y)=$ $\left(1+e^{-y}\right)^{-1}$ for Example 5 .

\section{References}

Ahsanullah, M. \& Wesolowski, J. (1998). Linearity of best predictors for non-adjacent record values. Sankhyā Ser. B 60, 221-227.

Arnold, B.C., Balakrishnan, N. \& Nagaraja, H.N. (1998). Records. New York: Wiley.

Dembinska, A. \& Wesolowski, J. (2000). Linearity of regression for non-adjacent record values. J. Statist. Plann. Inference 90, 195-205.

Franco, M. \& RUIZ, J.M. (1996). On characterization of continuous distributions by conditional expectation of record values. Sankhyā Ser. A 58, 135-141.

Nagaraja, H.N. (1977). On a characterization based on record values. Austral. J. Statist. 19, 70-73.

Nevzorov, V.B. (2001). Records: Mathematical Theory. Providence RI: American Mathematical Society.

Pakes, A.G. (2004). Product integration and characterization of probability laws. J. Appl. Statist. Sci. 13, $11-31$.

WesolowsKi, J. \& Ahsanullah, M. (1997). On characterizing distributions via linearity of regression for order statistics. Austral. J. Statist. 39, 69-78. 